\title{
Chapter 13 \\ The Role of the Teacher in Promoting Intercultural Approaches
}

\section{Introduction}

To complete this book with a chapter devoted to teachers is justified for several reasons. Firstly, the quality of a school is closely connected to the quality of its teachers. Their intellectual, moral and academic capacities are essential in our search for a more just and effective intercultural school. Then, our experience with different school systems encourages us to think that the school is more likely to introduce intercultural approaches when the attitude of the teachers combines "ethnocultural diversity seen as a problem to be overcome" with "ethnocultural diversity considered as an opportunity to teach and learn in another way and in a context valuing social justice". Only the teacher can decide on this change of attitude and of paradigm, but it is evident that training as well as the school itself have their part to play in offering the teachers the possibilities to pursue these indispensable changes.

Of course, a large number of teachers are tempted by the attitude "I do not pay attention to cultural or social difference" by asserting that they treat all children just the same. However, research has brought to light that teachers have perceptions, bias, expectations, choices and behaviours associated with their pupils' cultural origins.

Furthermore, the development of intercultural education should be based on a solid theoretical foundation that we have already dealt with in the first part of this book. Nevertheless, it would remain pointless if it were not accompanied in its implementation by the appropriate teaching methods and also forming a regular part of the school's daily life. The teachers are the principal agents in this process of educational transposition. Thus, by placing this responsibility on them, we plead in favour of reinforced initial and in-service training in this domain, as well as the contribution of specialists in reflecting on their journey towards diversity, difference or otherness and in the way they are employed in the classroom.

In this chapter, we will deal firstly with the need to change the way the teaching body interprets cultural diversity. Indeed, even if it would seem that the general attitude towards cultural difference is positive, some issues associated with the way the practitioners perceive it remain unresolved. In the second part, we will present 
different theoretical frameworks in which diversity is used as a resource and social justice becomes a principal objective of the school. These frames of reference represent resources on which teachers can base their intercultural teaching techniques. Finally, we will present recent work concerning the educational added-value deriving from the recruitment of teachers from diverse cultural backgrounds.

\section{Modifying Teachers' Perspectives on Language and Cultural Diversity}

One of our recent research projects has shown that teachers are in general open to diversity, seeking to accept it and at first glance not intending to avoid it (Radhouane, 2019). However, certain ways of looking at difference, multiculturality or at taking diversity into account lend themselves to confusion. For example, the question of identity is always ambivalent. One finds in some teachers' words elements indicating a stable conception of it, whereas the conversation of other teachers depicts an dynamic conception of it (Radhouane, 2019). We have also asked ourselves questions about the relevance of dubious expressions, such as "to be half-and-half" or "between two stools" when describing multiple affiliations (very often associated with double nationality or with a very diverse urban setting). This language aspect seems to betray an ambivalent attitude towards diversity, which we propose to examine here. Could these children who are labelled as "half-and-half" not be two wholes? Does their multiple identity have to be amalgamated to make just one? In the final analysis, does cultural identity have to be reduced to the mathematical unit of "one"? If one must actually consider identity as a coherent whole made up of several elements, this does not diminish the problems that multiple identities might lead to (misunderstandings between the school and the home, conflicts of values, etc.). Therefore, since culture is dynamic, it seems to us more relevant to recognize the plasticity of identity, the flexibility of affiliations, while, on the contrary, it seems counterproductive to want to "protect" children by limiting them to a single language and culture (Abdelilah-Bauer, 2015).

Some authors (Blaisdell, 2005; Sleeter and Grant, 2009; Boutte et al., 2011) have even used the term "colour-blindness" when considering the perception of the pupils' identities by the teacher. This term is employed to describe an attitude when the colour of the pupils' skin is not perceived and, by extension, neither is an individual's origin. Even if, according to Blaisdell (2005), very few teachers claim to assume this attitude, they often develop an egalitarian-style discourse (we are all equal) treating all pupils in the same way:

They view equality and fairness in treating their students exactly the same way regardless of racial background and the adherence to this view prevents these teachers from acting on the racial difference they see (Blaisdell, 2005, p. 34). 
Based no doubt on good intentions aimed at equal treatment, this attitude can however be detrimental, to the extent that intercultural education for social justice does require considering differences, since the pupils, according to their origins, the colour of their skin, the languages they speak, etc., do not all enjoy the same experience of the school. Obviously, there should be no temptation to fall into the opposite situation and to give cultural justifications to every one of the pupils' actions, very often in such situations resorting to a compensatory attitude resulting in a deviation, a failure and shortfall in relation to the educational expectations. There is, therefore, a real uncertainty between taking cultural origins into account, ignoring them and wildly exaggerating them. Examining this inconsistency is necessary when asking questions about the implementation of relevant intercultural approaches.

Then, we should come back to the effects of labelling of which certain pupils are the victims. Several studies, especially the famous Pygmalion in the classroom (Rosenthal \& Jacobson, 1968), ${ }^{1}$ have drawn attention to the effects of the way in which the teachers perceive their pupils' school performance: "I believe you are capable of succeeding and therefore I push you to succeed; on the contrary, if I believe you are heading for failure, (subconsciously) I push you towards it." Teachers must be familiar with this compact reformulation of what is known today as the "Pygmalion effect". Indeed, their appreciation of the pupils' potential may influence their expectations and therefore have an impact on their pupils' educational achievements. The study by McKown and Weinstein (2008) in the United States has shown that what is expected of children may, in some cases, depend upon their ethnic origins. It is therefore essential to be aware of the effects of labelling so as to counter them and in this way contribute towards a more just education.

Thus, the complexity of matters of identity, the impact of categorization, the failure to take the issues of cultural affiliation into account and the persistence of stereotypes in schools are among the reasons to insist upon the need to pursue efforts concerning the way teachers consider the diversity of languages and cultures.

\section{Working with Difference and Cultural Diversity: The Example of Culturally Relevant Teaching}

Cultural differences represent a challenge for the education system, particularly for the teachers. They work in a system that has historically been monocultural but which, despite its efforts towards openness, has to cope with the conservation of local/national unity and taking diversity into consideration through an integrative rather than an assimilationist approach. These tensions, typical of culturally diverse

\footnotetext{
${ }^{1}$ The name "Pygmalion" is taken from the legend bearing the same name. Pygmalion, the King of Cyprus, sculpted a statue with which he fell in love. The goddess of love transformed it into a woman. The traditional explanation is that it was the perception of this statue influenced by a feeling of love that gave rise to its transformation. It was therefore the expectation that foretold the outcome.
} 
education systems, come to the fore in the teacher's work. Thus, the question that we will attempt to answer here is: "How does one connect the values of openness and citizenship with sometimes heterogenous affiliations, beliefs and identities?"

The theory of culturally relevant teaching seems to be particularly useful in facing these tensions, while making them effective within the school. This conception of teaching, developed in the United States, is based on a critical vision of the world (Schmeichel, 2012). This pedagogy aims at three main objectives: the success of all pupils; the development of their cultural competence; and a critical approach to the existing social order (Ladson-Billings, 1995). Already, in this brief description, it is possible to appreciate that culturally relevant teaching is based on critical pedagogy and forms part of the resistance movements to oppression experienced by some groups within society. This critical conception allows diversity to be understood with all the complexity that it merits. Even when it is not considered as a problem, this does not mean that different cultural groups have not been the victims of various injustices. Culturally relevant teaching goes against the grain and proposes to deal with the problems experienced by cultural groups or individuals with diverse backgrounds rather than to treat these groups as problems in themselves.

Implementing culturally relevant teaching is based on different principles ranging from the recognition and legitimation of the pupils' cultures in the classroom to achieving the objectives of autonomy and social change (the pupils should be ready for role-playing) (Gay, 2010). Furthermore, it remains important to remember that this teaching has two facets. It is based on the theories of social justice for the pupils in order to combat unequal opportunities, potentials, school careers, etc., that might exist. But it is also rooted in social justice through the pupils themselves, since by the end of such teaching, they should be motivated agents working to reduce inequalities and ready to oppose various injustices (see Gay, 2010).

Moreover, Gay (2010) recommends the multidimensional implementation of culturally relevant teaching. For this purpose, the aspects mentioned previously must figure in all modes of teaching: evaluation; interactions between the pupils; with the teacher; with the parents; following the curriculum; classroom activities; etc. All of these aspects should, according to this theory, be influenced by a critical view of education, of inequality and therefore be employed in favour of greater justice in the school. Table 1 resumes the characteristics of teachers who apply the theory of culturally relevant teaching.

The interest of this theory is that it does not recommend miraculous solutions. It is based rather on existing educational objectives and practices that are exploited in the context of a recognition and a legitimation of the pupils' cultures and the struggle against inequalities. Finally, culturally relevant teaching is a useful framework for reflection on the organization of school work and interactions. For the teachers, it raises questions about their teaching methods rather than presenting a precise list of recommendations. 
Table 1 The qualities of teachers applying the theory of culturally relevant teaching

\begin{tabular}{l|l}
\hline Quality & Definition \\
\hline Socio-cultural awareness & $\begin{array}{l}\text { Is aware of the way in which socio-cultural } \\
\text { structures have an impact on individual } \\
\text { experiences and potential }\end{array}$ \\
\hline The intention of making a difference & $\begin{array}{l}\text { Consider themselves as change agents working } \\
\text { for more equity in the education system }\end{array}$ \\
\hline High expectations & $\begin{array}{l}\text { To have high expectations and demands for all } \\
\text { pupils, whatever their socio-cultural origin }\end{array}$ \\
\hline A constructive approach to teaching & $\begin{array}{l}\text { Understands that the learners construct their own } \\
\text { knowledge }\end{array}$ \\
\hline Profound understanding of the pupils & $\begin{array}{l}\text { Familiar with the lives of the pupils and their } \\
\text { families; know how the pupils learn best and their } \\
\text { present level of competence }\end{array}$ \\
\hline Practices culturally appropriate/relevant & $\begin{array}{l}\text { Constructs learning arrangements on the basis of } \\
\text { the pupils' present knowledge and augments their } \\
\text { thinking and knowledge }\end{array}$ \\
\hline
\end{tabular}

Source Adapted from Ontario Schools (2013)

\section{Cultural Diversity and Its Interactions}

Even though culturally relevant teaching tackles the question of inter-individual interactions, it seems necessary for us to examine this dimension more closely. Indeed, as we have seen previously, some teachers are tempted to adopt negative attitudes towards certain pupils from culturally diverse origins. However, these attitudes should be called into question so as to avoid setting in motion a vicious circle in the relationship between the teacher and the pupil or of perpetuating negative reinforcement as shown in Fig. 1. When confronted with labelling and the Pygmalion effect, the negative educational dynamic can quickly take hold and be difficult to reverse.

Given the harmful consequences of a negative educational situation, it seems to us indispensable to move towards a dynamic of positive reinforcement in the relationship between the teacher and the migrant pupil. This dynamic is illustrated in Fig. 2.

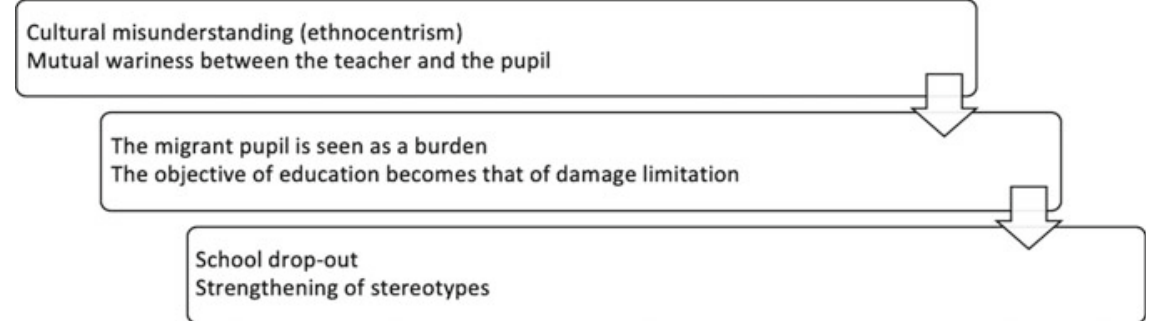

Fig. 1 The dynamic of negative reinforcement 


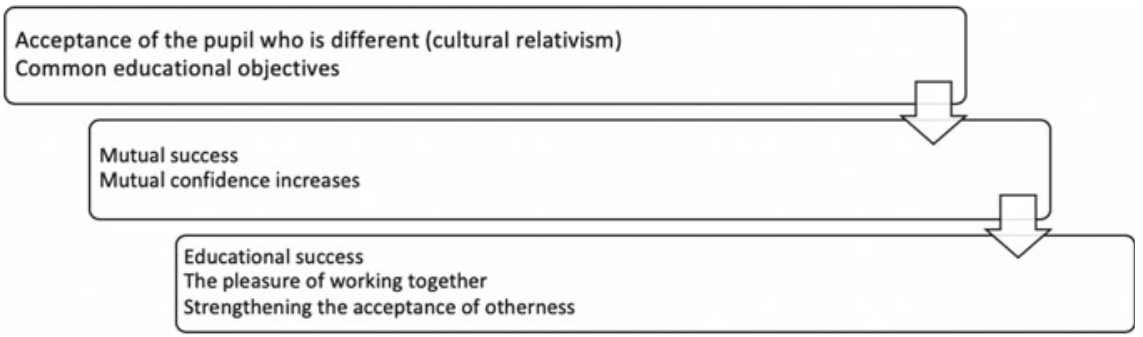

Fig. 2 The dynamic of positive reinforcement

It is, of course, the acceptance of cultural difference that differentiates these two figures. Educational professionals, even if they are shocked, dismayed or concerned about a cultural practice or a particular affiliation, should acknowledge their feelings so as to overcome them and not let them influence their teaching activities. It is natural to go through periods of uncertainty when faced with the unknown/otherness. However, awareness and an examination of one's own perceptions represent important steps in developing harmonious intercultural relationships.

On this subject, the work of Cohen-Emerique (2015) has demonstrated the different aspects of intercultural interaction. Firstly, she presents the professional (i.e. the teacher) as well as the Other (i.e. the pupil) as individuals distinguished by their culture, their religion, their social origins, etc. Secondly, she presents the reference framework for these two individuals (a professional reference framework for the teacher and an "Other" reference framework for the pupil). The interactions between these individuals and their reference frameworks are at the origin of an interface permitting negotiations and therefore an intercultural rapprochement. Three important aspects should be borne in mind concerning this approach developed by Cohen-Emerique (2015): decentration, required by professionals wishing to understand the attitude of an Other who is different from them; the desire to enter into the Other's system, required by the pupil to multiply interactions; and, lastly, the context. Indeed, in an intercultural relationship, Cohen-Emerique (2015) lays stress on the need to take into account the individuals concerned: are they in a dominant/dominated relationship? former colonizer/former colonized? etc.

The approach proposed by Cohen-Emerique (2015) seems very interesting to us inasmuch as it allows the relationship between a teacher and a migrant pupil to be analysed. However, it should be observed that the reference frameworks of the two protagonists may be ambiguous and hybrid. For instance, teachers may remain faithful to the reference framework of their profession but, at the same time, come from mixed-race ethno-cultural origins. Meanwhile, the migrant pupils are influenced by the reference framework of their family and their country of origin. Nevertheless, they may choose to distance themselves from this reference framework. For example, adolescent migrant pupils may resist a traditional practice from their culture of origin in order to follow another reference framework. 
According to Lemoine (2018), the concept "intercultural" consists of four elements of foreignness/otherness concerning languages or other matters. The first element is the relationship with foreigners; the second element would be the relationship with classmates conducted in the teaching language; the third element concerns the relationship with oneself_-not only understanding oneself, but asking oneself about the attitudes that $I$ have towards others; finally, the fourth element concerns the situations proposed by the teacher using stereotypical cultural content. These four elements could be an interesting avenue of reflective thinking for professionals wishing to understand the approach to otherness and the relationships that develop in an intercultural context.

\section{The Added-Value of Teachers from Diverse Backgrounds}

Repeatedly, research on intercultural education has deplored the lack of diversity within the teaching body (Akkari, 2003). The basic idea is that the pupils could relate to adults that resembled them (in other words, shared similar experiences concerning their multiple cultural affiliations) and that their identities could be accepted and recognized within the school. For these reasons, research on teachers with culturally diverse backgrounds has expanded considerably, particularly in the United States, then in the remainder of the English-speaking world, and subsequently in the Frenchspeaking world (see, for example, Hess \& Leal, 1997; Cherng \& Halpin, 2016; Atkins, Fertig \& Wilkins, 2014).

The general hypothesis for this field of research is that teachers with a diverse culture/origin/ethnicity compared to that of the school in which they are teaching are likely to bring an educational added-value, especially when this school serves an ethnically diverse population. We will examine this hypothesis from different angles: firstly, what motivated these teachers to enter this profession; and secondly, the benefits of their identity to their teaching practice.

In the first instance, it is necessary to dwell on the professional motivations for this type of teacher. If they differ in the way they entered the teaching profession, it is perhaps because these teachers with culturally diverse origins were not always motivated by the same reasons as their peers from the dominant group. Their potential familiarity with the pupils' experiences (Magaldi et al., 2018) or their own experiences with schooling troubled by discrimination and racism $(\mathrm{Su}, 1997)$ may have had an impact on the way some of them conceive their role as teacher. In contrast with their peers belonging to the majority group, they are as a rule more familiar with the issues associated with social justice and inequality experienced by poor or minority populations (Su, 1997; Magaldi et al., 2018). Some of them express reproaches about the inequalities of educational opportunities, the lack of relevance of the curricula or the way pupils with culturally diverse backgrounds are taught (Su, 1997). It was as a reaction to these shortcomings that they chose the teaching profession so as to 
become change agents in their pupils' lives (Su, 1997; Magaldi et al., 2018) but also in society ( $\mathrm{Su}, 1997)$. Some of these teachers, inspired by their own experiences, demonstrate the resilience that they have acquired from it and are therefore more likely to transform their personal experiences into educational resources of benefit for their pupils.

A Swiss research project has also shown that some young people from diverse origins may choose the teaching profession as a way of countering "identity assignation" (Changkakoti \& Broyon, 2013, p. 104); in other words, they wanted to demonstrate their capacity to succeed despite the stereotypes attached to their culture of origin.

In the second approach, it was a question of analysing the effect that the identities of the teachers with culturally diverse origins might have on their teaching practice. On this matter, Villegas and Irvine (2010) made a list of several studies that showed the positive impact that these teachers could have on the educational achievement of pupils belonging to cultural minorities. One could believe that their resort to culturally relevant teaching (Villegas \& Irvine, 2010) was at the origin of their pupil's proven success.

Moreover, in the classroom, these teachers will be more susceptible to understanding the pupils' experiences and may develop a feeling of empathy for them (Magaldi et al., 2018).

In their words, one can discern that the educational institution is kept at arm's length. In their eyes, it is too ethnocentric and does not take the pupils' cultural origins into account. The teachers questioned by Kohli (2009) challenged the invisibility of their cultures in the curriculum:

By prioritizing European history, and ignoring or tokenizing the contributions and experiences of non-White people, Kimmy's social studies class affirmed a racial hierarchy. Subtle or not, she was sent the message that White culture and history are important and that the identity, culture and the history of her people are unimportant (Kohli, 2009, p. 242).

The author explains next that this acquaintance with the racism and discrimination present in educational institutions that teachers with culturally diverse origins may have experienced when young could be a force to be exploited during their training (Kohli, 2009). This would be of considerable benefit to those pupils from similar culturally diverse origins. In fact, one could imagine that these experiences create an understanding with the pupils and strengthen the sentiment of empathy that these teachers may possess (Magaldi et al., 2018).

The added-value of teachers with culturally diverse origins is also present in their teaching practice. Firstly, they are most likely to teach in a culturally relevant manner (Villegas \& Irvine, 2010). In this way they may be able to create links between the school and the home, which may contribute to strengthening the meaning of learning.

Secondly, various studies have shown a correlation between the presence of teachers with culturally diverse origins and the outcomes of their educational performance (Pitts, 2007; Villegas \& Irvine, 2010; Farkas et al., 1990). The study by Pitts 
(2007) brought to light a statistically significant link between teachers with culturally diverse origins and the educational achievement of pupils from cultural minorities. Farkas et al. (1990) have shown not an impact on the pupils' school marks but rather on their working habits. A teacher with diverse origins could have an influence on them and also contribute to academic achievement. In general, the scientific literature draws attention to a trend among these teachers who have higher expectations with regard to all of their pupils (Villegas \& Irvine, 2010).

Thirdly, Meire (1993, quoted by Villegas \& Irvine, 2010) has observed that the numbers of pupils redirected to special education drop when teachers of diverse origins are more numerous in the school.

To summarize, Villegas and Irivine (2010) identified a list of five characteristics of these teachers:

1. They have higher expectations with regard to all pupils;

2. They employ a culturally relevant pedagogy;

3. They establish positive relationships based on care and confidence in their pupils;

4. They become involved in problems connected with racism;

5. They defend the pupils' interests (Villegas \& Irvine, 2010, quoted by Radhouane, 2019).

Thus, there are numerous positive outcomes resulting from the presence of teachers with diverse cultural origins. However, the reader should beware of two important factors. Firstly, the majority of the studies mentioned are taken from English-speaking contexts and may have been influenced by this context. Secondly, we have drawn attention here to trends that were often the outcomes of qualitative research. It is necessary in this case to consider the flexibility and the diversity that may include "teachers with culturally diverse origins". Furthermore, the research by Radhouane (2019) shows that within the sample, even when statistically adjusted, not all teachers with culturally diverse origins corresponded to the features established here and that disparities associated with personal experiences as well as their identification with a professional identity existed. From this same research, the study of literature on teachers with diverse cultural origins allowed three reservations to be identified when working on this theme: firstly, the group of "teachers with culturally diverse origins" is not homogenous; secondly, one should not award these teachers responsibilities that go beyond their functions (to diminish all the inequalities at the school); and thirdly, one should not neglect the intercultural competencies of teachers who are neither of migratory origins nor from ethnocultural minorities (Radhouane, 2019). ${ }^{2}$ One should then adopt a relativist attitude typical of intercultural approaches, as well as developing a dynamic conception of identity allowing teachers with diverse cultural origins either to identify themselves with this category or not and therefore to remain entirely responsible for the definition of their own identity.

\footnotetext{
2 The author draws largely on the works of Bressler and Rotter (2017), Santoro (2016), Legendre (2004) and Georgi (2016).
} 


\section{Conclusion}

Today, it would be an exaggeration to say that cultural diversity is perceived as a problem within education systems, since the teachers, in general, accept it and have positive attitudes towards the contribution of diversity. However, even if it is not a problem within the school, one must nevertheless address its complexity. Cultural diversity is not only an auspicious resource for extra-curricular activities; it has a social dimension with an impact on the life of the pupils and their parents. From this point of view, it seems important to us that teachers are aware of the issues concerning such themes as integration, openness, social justice and equality. Otherwise, it should be said that a certain number of stereotypes persist and their effects should be thoroughly examined within the school.

One of the theoretical foundations which seems productive for us in promoting cultural diversity is culturally relevant teaching. Indeed, further to exploiting otherness as a resource, it subjects it to critical analysis and in this way addresses inequalities connected with cultural, social, ethnic, religious or sexual affiliations. This form of teaching addresses inequalities, but also takes the form of an approach to combat them.

Finally, research has demonstrated the advantage of opening the teaching profession to people from diverse origins. Indeed, their knowledge and the resemblance between their own experiences and those of their pupils, particularly those with cultural minority origins, contribute an educational added-value. Furthermore, we believe that the experiences of these teachers may represent a resource for the educational enterprise, while accepting its limits: the need for each individual to choose their own affiliation and not to be assigned an identity that could be detrimental.

Open Access This chapter is licensed under the terms of the Creative Commons Attribution 4.0 International License (http://creativecommons.org/licenses/by/4.0/), which permits use, sharing, adaptation, distribution and reproduction in any medium or format, as long as you give appropriate credit to the original author(s) and the source, provide a link to the Creative Commons license and indicate if changes were made.

The images or other third party material in this chapter are included in the chapter's Creative Commons license, unless indicated otherwise in a credit line to the material. If material is not included in the chapter's Creative Commons license and your intended use is not permitted by statutory regulation or exceeds the permitted use, you will need to obtain permission directly from the copyright holder.

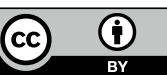

